\title{
Influência da Palha e da Simulação de Chuva sobre a Eficácia da Mistura Formulada Clomazone + Hexazinone no Controle de Plantas daninhas em Área de Cana-Crua ${ }^{1}$
}

\author{
Effect of Sugarcane Straw and Rain Simulation on the Efficacy of Clomazone + Hexazinone in \\ Controlling Weeds in Raw Sugarcane Area
}

\author{
NEGRISOLI, E. ${ }^{2}$, VELINI, E.D. ${ }^{3}$, CORREA, M.R.. ${ }^{4}$, ROSSI, C.V.S. ${ }^{5}$, CARBONARI, C.A. ${ }^{6}$, \\ COSTA, A.G.F. ${ }^{7}$ e PERIM, L. ${ }^{8}$
}

\begin{abstract}
RESUMO - O experimento teve o objetivo de avaliar os efeitos da cobertura de palha e da simulação de chuva sobre a eficácia da mistura formulada clomazone + hexazinone no controle de plantas daninhas em área de cana-crua. Foi avaliado o controle de Brachiaria decumbens, Ipomoea grandifolia, Ipomoea hederifolia e Euphorbia heterophylla. A dose do herbicida utilizada foi de $2,2 \mathrm{~kg} \mathrm{ha}^{-1}$ de produto comercial, correspondendo a 880 e $220 \mathrm{~g} \mathrm{ha}^{-1}$ dos ingredientes ativos clomazone e hexazinone, respectivamente. Os tratamentos utilizados foram: T1) semeadura + palha 5 t ha-1 + aplicação + chuva de $30 \mathrm{~mm}$ (1DAA); T2) semeadura + chuva de $30 \mathrm{~mm}$ + palha $5 \mathrm{t} \mathrm{ha}^{-1}$ + aplicação; T3) semeadura + aplicação + palha $5 \mathrm{t}$ ha ${ }^{-1}$; T4) semeadura + palha $5 \mathrm{t} \mathrm{ha}^{-1}+$ chuva de $30 \mathrm{~mm}$ + aplicação (12h após); T5) semeadura + palha $5 \mathrm{t} \mathrm{ha}^{-1}+$ aplicação + chuva de 2,5 mm (logo após); T6) semeadura + aplicação + chuva de $30 \mathrm{~mm}$; T7) testemunha sem palha; e T8) testemunha com 5 t ha ${ }^{-1}$ de palha, totalizando oito tratamentos com quatro repetições, dispostos no delineamento experimental de blocos casualizados. Foram feitas avaliações visuais de controle aos 6, 13, 21, 27 e 35 dias após a aplicação (DAA). Para controle de $B$. decumbens, os melhores tratamentos foram aqueles nos quais o herbicida foi aplicado diretamente no solo, recebendo ou não uma camada de palha sobre o solo após a aplicação do herbicida, e quando foi aplicado sobre a camada de palha, recebendo uma chuva após a aplicação. Para a espécie E. heterophylla, os resultados foram bastante satisfatórios, proporcionando médias acima de $98 \%$ de controle, quando ocorreram precipitações posteriores à aplicação do herbicida. De modo geral, os tratamentos com a aplicação do herbicida, na ausência ou presença de palha, e posterior chuva apresentaram controle total da espécie I. hederifolia aos 35 DAA. Todos os tratamentos mostraram excelente controle para a espécie I. grandifolia.
\end{abstract}

Palavras-chave: Saccharum spp., cana-de-açúcar, herbicidas.

\begin{abstract}
The experiment was conducted to evaluate the efficacy ofcombining clomazone + hexazinone and straw cover to control weeds in raw sugarcane area. The species Brachiaria decumbens, Ipomoea grandifolia, Ipomoea hederifolia and Euphorbia heterophylla were evaluated. The herbicide rate used was $2.2 \mathrm{~kg} \mathrm{ha}^{-1}$ of the commercial product, corresponding to 880 and $220 \mathrm{~g} \mathrm{ha}^{-1}$ of the active ingredients, clomazone and hexazinone, respectively. The treatments were: $T 1$ ) seeding + $5 \mathrm{t}$ ha-1 of straw + application + rain $30 \mathrm{~mm}(1 \mathrm{DAA}) ; \mathrm{T} 2)$ seeding + rain (30 mm) + $5 t$ ha $^{-1}$ of straw + application; T3) seeding + application $+5 t$ ha $^{-1}$ of straw; T4) seeding $+5 t$ ha $^{-1}$

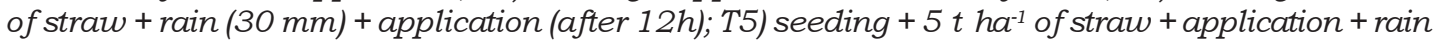
$2.5 \mathrm{~mm}$ (after application); T6) seeding + application + rain $(30 \mathrm{~mm}) ;$ T7) control without straw, and
\end{abstract}

1 Recebido para publicação em 15.5.2010 e na forma revisada em 18.2.2011.

2 Engo-Agro ${ }^{\circ}$, Dr., TechField-Nupam - Faculdade de Ciências Agronômicas, Universidade Estadual Paulisa "Júlio Mesquita Filho" -

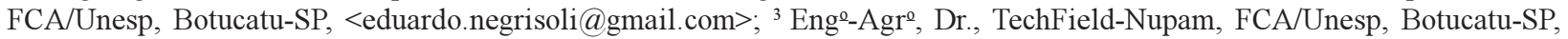
<marcelorcorrea@uol.com.br>; ${ }^{4}$ Professor Adjunto, Dr., Dep. de Agricultura, FCA/Unesp, Fazenda Experimental Lageado,

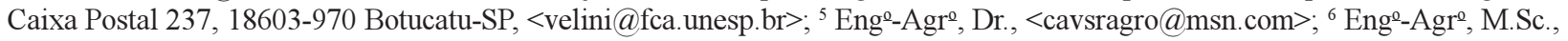
FCA/Unesp, Botucatu-SP, <carbonari@fca.unesp.br>; ${ }^{7}$ Engo-Agr ${ }^{\circ}$, Dr., <augustogfcosta@hotmail.com>; ${ }^{8}$ Aluno de Mestrado, FCA/Unesp, Botucatu-SP, <lperim@fca.unesp.br.

Planta Daninha, Viçosa-MG, v. 29, n. 1, p. 169-177, 2011 
T8) control with $5 t$ ha ${ }^{-1}$ straw, totaling eight treatments with four replications in a randomized block design. A visual control evaluation was carried out at 6, 13, 21, 27 and 35 days after application (DAA). For the species B. decumbens, the best treatments observed were when the herbicide was applied directly on the soil, or not receiving a layer of straw on the ground and when it was applied on the layer of straw, with rainfall following application. For the species $\boldsymbol{E}$. heterophylla, the results were quite satisfactory, providing averages of $98 \%$ of control, when rainfall occurred after herbicide application. In general, herbicide treatments in the absence or presence of straw, and subsequent rainfall and/or soil moisture, provided a full control of I. hederifolia, at 35 DAA. All treatments showed excellent control of the species I. grandifolia.

Keywords: Saccharum spp., sugarcane, herbicides.

\section{INTRODUÇÃO}

Dentre os fatores bióticos, as plantas daninhas são um dos principais componentes do agroecossistema da cana-de-açúcar que interferem no desenvolvimento e na produtividade dessa cultura. A presença dessas plantas pode interferir no processo produtivo da cana-deaçúcar, competindo pelos recursos do meio, principalmente água, luz e nutrientes, liberando substâncias alelopáticas, atuando como hospedeiro de pragas e doenças comuns à cultura e interferindo nas práticas de colheita. A ocorrência de um ou mais desses componentes de interferência poderá reduzir a quantidade de colmos colhidos e diminuir o número de cortes economicamente viáveis. Essas interferências estão amplamente relatadas em diversos artigos científicos (Durigan et al., 2006; Kuva et al., 2007; Oliveira \& Freitas, 2008).

A palha é apenas uma das barreiras para o uso de herbicidas com ação exclusiva ou preferencial no solo. O acréscimo do teor superficial de matéria orgânica no solo, menos evidente em cana-crua do que em plantio direto, em razão da movimentação mínima no momento da colheita mecanizada associada ao preparo e sulcamento quando da reimplantação da cultura, propicia a adsorção dos herbicidas, limitando a sua eficiência (Negrisoli et al., 2005). Algumas plantas daninhas, como Ipomoea grandifolia e Euphorbia heterophylla, não têm sua germinação inibida pelas quantidades de palha de cana-de-açúcar que normalmente são encontradas em campo (Martins et al., 1999; Velini \& Negrisoli, 2000; Negrisoli et al., 2007a).

A cobertura morta ocasiona mudanças químicas, físicas e biológicas no solo, podendo provocar seleção da comunidade infestante e suprimir a infestação de plantas daninhas normalmente consideradas importantes nos canaviais, como Digitaria horizontalis, Brachiaria plantaginea, Brachiaria decumbens e Panicum maximum (Gravena et al., 2004). No entanto, espécies adaptadas ao sistema de cana-crua, como Euphorbia heterophylla e Ipomoea grandifolia, estão cada vez mais presentes nos canaviais (Martins et al., 1999; Correia \& Durigan, 2004).

As convolvuláceas, principalmente as pertencentes aos gêneros Ipomoea e Merremia, destacam-se entre as plantas daninhas que podem causar sérios danos à cultura da canade-açúcar, especialmente em áreas de canacrua. Além de competirem com a planta cultivada, podem interferir nas práticas culturais, especialmente na colheita mecanizada, reduzindo sua eficiência (Azania et al., 2002). Algumas plantas daninhas, como I. grandifolia e E. heterophylla, não têm a germinação inibida pelas quantidades de palha de cana-deaçúcar normalmente encontradas em campo (Martins et al., 1999; Gravena et al., 2004; Negrisoli et al., 2009).

Correia \& Durigan (2004) estudaram os efeitos da cobertura do solo, com 0, 5, 10 e $15 \mathrm{t} \mathrm{ha}^{-1}$, sobre a emergência de seis espécies de plantas daninhas (B. decumbens, D. horizontalis, Sida spinosa, I. grandifolia, Ipomoea hederifolia e Ipomoea quamoclit) e constataram que a cobertura do solo com 5, 10 e $15 \mathrm{t} \mathrm{ha}^{-1}$ inibiu a emergência de plântulas das espécies $B$. decumbens e $S$. spinosa, sendo o mesmo observado para $D$. horizontalis submetida a 10 e $15 \mathrm{tha}^{-1}$. No entanto, para I. grandifolia e I. hederifolia, o número de plantas emergidas não diferiu entre as quantidades de palha. Por sua vez, a presença da 
cobertura morta incrementou a emergência de plântulas de I. quamoclit.

A mistura clomazone thexazinone é indicada para aplicações em pré-emergência na cultura da cana-de-açúcar, na época de soca-seca, em periodos de baixos indices pluviométricos, principalmente durante os meses de inverno no Brasil Central. A solubilidade do clomazone em água é de $1.100 \mathrm{mg} \mathrm{L}^{-1}$, e a do hexazinone, de $32.000 \mathrm{mg} \mathrm{L}^{-1}$ (Rodrigues \& Almeida, 2005).

Ainda segundo Rodrigues \& Almeida (2005), o clomazone é um herbicida do grupo das isoxazolidinonas, inibe a sintese de carotenos em tecidos vegetais e, apesar da solubilidade relativamente alta em água, é adsorvido por coloides em proporções relativamente elevadas, razão pela qual apresenta baixa lixiviação no perfil do solo. Por outro lado, esses autores também relatam que o hexazinone é do grupo das triazinas, atua sobre o fotossistema II e apresenta alta mobilidade no solo com elevado potencial de lixiviação.

A lixiviação de herbicidas no perfil do solo sofre forte influência da quantidade e da época de ocorrência de chuvas após a aplicação do produto, como já constatado para metribuzin (Banks \& Robinson, 1982), oryzalin (Banks \& Robinson, 1984) e alachlor e metolachlor (Banks \& Robinson, 1986). Além do regime pluvial, da adsorção aos coloides e da taxa de infiltração de água no solo, a intensidade de lixiviação de herbicidas é fortemente dependente das características físico-químicas dos produtos aplicados, principalmente da solubilidade em água (Deuber, 1992).

Lamoreaux et al. (1993) ressaltam que a lavagem de herbicidas da palha para o solo é dependente da capacidade desses resíduos de cobrir o solo e reter os herbicidas; da solubilidade do produto aplicado; e do período em que a área permanece sem chuva após a aplicação do produto.
Este trabalho teve como objetivo avaliar os efeitos da cobertura de palha e da simulação de chuva sobre a eficácia da mistura formulada clomazone + hexazinone, no controle de plantas daninhas em área de cana-crua.

\section{MATERIAL E MÉTODOS}

A presente pesquisa foi realizada em condições controladas de casa de vegetação no Núcleo de Pesquisas Avançadas em Matologia (NUPAM), pertencente ao Departamento de Produção Vegetal da Faculdade de Ciências Agronômicas/UNESP - Botucatu-SP.

O solo utilizado foi inicialmente seco à sombra por um período de $48 \mathrm{~h}$. Após esse período, foi passado em peneira com malha de 200 mesh e submetido às análises químicas e granulométricas (Tabelas 1 e 2). Com base nesses resultados, o solo foi adubado para propiciar melhor desenvolvimento das plantas daninhas e, em seguida, acondicionado em vasos de $2 \mathrm{~L}$ de capacidade, procedendo-se à semeadura com as espécies Brachiaria decumbens, Ipomoea grandifolia, Ipomoea hederifolia e Euphorbia heterophylla.

A umidade do solo, medida gravimetricamente, foi mantida durante todo o ensaio em $65 \%$ da capacidade de campo.

A pulverização do herbicida e a simulação de chuva foram realizadas através de um equipamento instalado em laboratório do NUPAM, o qual é constituído de uma estrutura metálica com $3 \mathrm{~m}$ de altura por $2 \mathrm{~m}$ de largura, que permite acoplamento de um "carrinho" suspenso a 2,5 m de altura. A esse carrinho encontramse acopladas duas barras de pulverização: uma responsável pelo sistema de simulação de chuva e a outra pelo sistema de pulverização de defensivos agrícolas, as quais se deslocam por uma área útil de $6 \mathrm{~m}^{2}$ no sentido do comprimento do equipamento. O tracionamento de ambas as barras é realizado por meio de

Tabela 1 - Análise química da amostra de solo utilizado nos vasos. Botucatu-SP, 2007

\begin{tabular}{|c|c|c|c|c|c|c|c|c|c|c|c|}
\hline \multirow{2}{*}{ Solo } & $\mathrm{pH}$ & $\mathrm{MO}$ & P res. & $\mathrm{K}^{+}$ & $\mathrm{Ca}^{+2}$ & $\mathrm{Mg}^{+2}$ & $\mathrm{H}^{+}+\mathrm{Al}^{+3}$ & $\mathrm{SB}$ & $\mathrm{T}$ & $\mathrm{V}$ \\
\cline { 2 - 11 } & $\left(\mathrm{CaCl}_{2}\right)$ & $\left(\mathrm{g} \mathrm{dm}^{-3}\right)$ & $\left.(\mathrm{mg} \mathrm{dm})^{-3}\right)$ & \multicolumn{5}{|c|}{$\left(\mathrm{mmol}_{\mathrm{c}} \mathrm{dm}^{-3}\right)$} & $(\%)$ \\
\hline LVd & 4,3 & 19 & 1 & 0,6 & 10 & 4 & 58 & 14,6 & 73 & 21 \\
\hline
\end{tabular}

Laboratório de Fertilidade do Solo, Departamento de Solos, Faculdade de Ciências Agronômicas/UNESP - Botucatu-SP. 
correntes e engrenagens, com auxílio de um motor elétrico, cujo ajuste é dado por um modulador de frequência, permitindo a obtenção de velocidade constante previamente determinada. O sistema de pulverização tem funcionamento independente do sistema de simulação de chuva, apesar de ambos compartilharem o mesmo equipamento e as mesmas funções de controle.

A simulação da chuva foi realizada utilizando-se uma bomba hidráulica de pressão constante e acionamento automático, a qual bombeia água armazenada de um reservatório com capacidade para $1.000 \mathrm{~L}$ até a barra e pontas de pulverização responsáveis pela formação de gotas de chuva. A barra de simulação de chuva situada a 1,45 m de altura em relação à superficie das unidades experimentais é constituída por três bicos de pulverização TK-SS-20 de alta vazão, espaçados de $0,5 \mathrm{~m} \mathrm{e}$ posicionados de forma a propiciar maior uniformidade de precipitação na área aplicada.

Esse sistema foi operado com velocidade de deslocamento de $0,187 \mathrm{~km} \mathrm{~h}^{-1}$, o que correspondeu a 2,5 Hertz no modulador de frequência e pressão de trabalho de $0,81 \mathrm{kgf} \mathrm{s}^{-2}$. Cada lâmina aplicada correspondeu a aproximadamente 2,5 mm de chuva. Essas especificações proporcionaram a produção de gotas artificiais de chuva com diâmetro mediano volumétrico (DMV) de 1.140 micras, conforme informações do fabricante da ponta de pulverização (Spraying Systems Co.).

A barra de pulverização é constituída por quatro pontas de pulverização XR 11002 VS, espaçadas de $0,5 \mathrm{~m}$ e posicionadas a $0,5 \mathrm{~m}$ de altura em relação à superfície das unidades experimentais. Para as pulverizações do herbicida, o sistema foi operado com velocidade de deslocamento de $3,6 \mathrm{~km} \mathrm{~h}^{-1}$, o que correspondeu a 45,0 Hertz no modulador de frequência, com consumo de calda correspondente a $200 \mathrm{~L} \mathrm{ha}^{-1}$. O equipamento foi operado sob pressão constante de 1,5 bar, pressurizado por ar comprimido. A pulverização do herbicida foi realizada na dose de $2,2 \mathrm{~kg} \mathrm{ha}^{-1}$ de produto comercial (Ranger $\left.{ }^{\circledR}\right)$, correspondendo a 880 e $220 \mathrm{~g} \mathrm{ha}^{-1}$, dos ingredientes ativos clomazone e hexazinone, respectivamente.

Na Tabela 3 encontram-se os tratamentos utilizados no experimento conduzido em vasos com diferentes posicionamento.s

Tabela 2 - Análise granulométrica do solo utilizado no experimento. Botucatu-SP, 2007

\begin{tabular}{|c|c|c|c|c|c|c|c|}
\hline \multirow{2}{*}{ Granulometria (\%) } & \multicolumn{9}{|c|}{ Classe de solo } & \multirow{2}{*}{ Classe textural } \\
\cline { 2 - 7 } & \multirow{2}{*}{ Argila } & \multirow{2}{*}{ Silte } & \multicolumn{5}{|c|}{ Areia } \\
\cline { 5 - 8 } & & & Fina & Média & Grossa & Total & \multirow{2}{*}{ Média } \\
\hline \multirow{2}{*}{ LVd } & 20 & 4 & 22,9 & 35,7 & 17,4 & 76 & 76 \\
\hline
\end{tabular}

Laboratório de Fertilidade do Solo, Departamento de Solos, Faculdade de Ciências Agronômicas/UNESP - Botucatu-SP.

Tabela 3 - Descrição dos tratamentos utilizados no experimento de eficácia da mistura formulada de clomazone + hexazinone, aplicada em diferentes posicionamentos em relação à palha de cana-de-açúcar e à simulação de chuva. Botucatu-SP, 2007

\begin{tabular}{|c|l|}
\hline Tratamento & \multicolumn{1}{|c|}{ Ordem sequencial de condução } \\
\hline 1 & Semeadura + palha $5 \mathrm{t} \mathrm{ha}^{-1}+$ aplicação + chuva de $30 \mathrm{~mm}$ (1DAA) \\
\hline 2 & Semeadura + chuva de $30 \mathrm{~mm}+$ palha $5 \mathrm{t} \mathrm{ha}^{-1}+$ aplicação \\
\hline 3 & Semeadura + aplicação + palha $5 \mathrm{t} \mathrm{ha}^{-1}$ \\
\hline 4 & Semeadura + palha $5 \mathrm{t} \mathrm{ha}^{-1}+$ chuva de $30 \mathrm{~mm}+$ aplicação $(12 \mathrm{~h}$ após $)$ \\
\hline 6 & Semeadura + palha $5 \mathrm{t} \mathrm{ha}^{-1}+$ aplicação + chuva de $2,5 \mathrm{~mm}$ (logo após) \\
\hline 7 & Semeadura + aplicação + chuva de $30 \mathrm{~mm}$ \\
\hline 8 & Semeadura + chuva de $30 \mathrm{~mm}$ \\
\hline
\end{tabular}

Obs.: aplicação de 880 e $220 \mathrm{~g} \mathrm{ha}^{-1}$, dos ingredientes ativos clomazone e hexazinone, respectivamente. 
O controle das espécies de plantas daninhas foi determinado por meio de uma escala visual e percentual de notas, variando de zero a 100. Nesse caso, zero consistia em nenhum controle e 100 era a porcentagem de controle total das espécies daninhas. As avaliações foram realizadas até o desaparecimento ou a estabilização dos sintomas de fitointoxicação (SBCPD, 1995). As avaliações visuais de controle foram realizadas aos 6, 13, 21, 27 e 35 dias após a aplicação (DAA), sendo também correlacionadas com a escala de notas da Asociación LatinoAmericana de Malezas (ALAM, 1974), apresentada na Tabela 4, a qual varia de 1 (nenhum a pobre) a 6 (excelente controle). O delineamento experimental foi o de blocos casualizados, e os dados foram comparados estatisticamente pelo teste de Tukey a $5 \%$ de probabilidade.

Tabela 4 - Escala de notas da ALAM utilizada para avaliação da eficácia de controle de plantas daninhas

\begin{tabular}{|c|l|}
\hline Porcentagem (\%) & \multicolumn{1}{|c|}{ Grau de controle } \\
\hline $0-40$ & Nenhum a pobre (1) \\
\hline $41-60$ & Regular (2) \\
\hline $61-70$ & Suficiente (3) \\
\hline $71-80$ & Bom (4) \\
\hline $81-90$ & Muito Bom (5) \\
\hline $91-100$ & Excelente (6) \\
\hline
\end{tabular}

\section{RESULTADOS E DISCUSSÃO}

Os resultados da eficácia de controle da mistura formulada de clomazone + hexazinone associada à cobertura de palha e à simulação de chuva estão apresentados nas Tabelas 5 a 8, conforme a espécie avaliada.

Para a espécie $B$. decumbens (Tabela 5), os melhores tratamentos observados foram aqueles nos quais se aplicou o herbicida diretamente no solo, recebendo (tratamento 3) ou não (tratamento 6) uma camada de palha de $5 \mathrm{t} \mathrm{ha}^{-1}$ sobre o solo. Esses tratamentos apresentaram excelente controle (nota $6 ; 100 \%$ ) aos 35 dias após a aplicação (DAA), com a presença de palha e 99,3\% (nota 6), quando de sua ausência nas unidades experimentais. Além disso, os tratamentos em que o herbicida foi aplicado sobre a camada de palha, recebendo uma chuva de $30 \mathrm{~mm}$ (tratamento 1) e $2,5 \mathrm{~mm}$ (tratamento 5) após a aplicação, também apresentaram excelentes resultados desde o início das avaliações. Resultados semelhantes foram encontrados por Negrisoli et al. (2007a,b), porém testando outros herbicidas (tebuthiuron e amicarbazone), nos quais os melhores resultados de controle foram encontrados quando os herbicidas foram aplicados diretamente no solo e/ou recebendo uma chuva após a aplicação do produto sobre a superfície com palha.

Tabela 5 - Porcentagem média e notas segundo a escala da ALAM de controle de Brachiaria decumbens pela mistura formulada de clomazone + hexazinone, aplicada em diferentes posicionamentos em relação à palha de cana-de-açúcar e à simulação de chuva. Botucatu-SP, 2007

\begin{tabular}{|c|c|c|c|c|c|c|c|c|c|c|}
\hline \multirow{3}{*}{ Tratamento } & \multicolumn{10}{|c|}{ Dias após a aplicação } \\
\hline & \multicolumn{2}{|c|}{6} & \multicolumn{2}{|c|}{13} & \multicolumn{2}{|c|}{21} & \multicolumn{2}{|c|}{27} & \multicolumn{2}{|c|}{35} \\
\hline & $\%$ & ALAM & $\%$ & ALAM & $\%$ & ALAM & $\%$ & ALAM & $\%$ & ALAM \\
\hline 1. P/A/chuva (30 mm - 1 DAA $)$ & $87,8 \mathrm{a}$ & 5 & $98,8 \mathrm{a}$ & 6 & $99,8 \mathrm{a}$ & 6 & $100,0 \mathrm{a}$ & 6 & $100,0 \mathrm{a}$ & 6 \\
\hline 2. Chuva $(30 \mathrm{~mm}) / \mathrm{P} / \mathrm{A}$ & $36,3 \mathrm{~b}$ & 1 & $71,3 \mathrm{c}$ & 4 & $83,3 \mathrm{~b}$ & 5 & $83,3 \mathrm{bc}$ & 5 & $57,5 \mathrm{~b}$ & 2 \\
\hline 3. A/P & $98,8 \mathrm{a}$ & 6 & $99,3 \mathrm{a}$ & 6 & $100,0 \mathrm{a}$ & 6 & $100,0 \mathrm{a}$ & 6 & $100,0 \mathrm{a}$ & 6 \\
\hline 4. $\mathrm{P} /$ chuva $(30 \mathrm{~mm}) / \mathrm{A}(12 \mathrm{~h}$ após $)$ & $15,0 \mathrm{c}$ & 1 & $82,0 \mathrm{bc}$ & 5 & $88,3 \mathrm{~b}$ & 5 & $78,3 \mathrm{c}$ & 4 & $55,0 \mathrm{~b}$ & 2 \\
\hline 5. P/A/chuva $(2,5 \mathrm{~mm})$ & $100,00 \mathrm{a}$ & 6 & $91,5 \mathrm{ab}$ & 6 & $97,8 \mathrm{a}$ & 6 & $93,8 \mathrm{ab}$ & 6 & $100,0 \mathrm{a}$ & 6 \\
\hline 6. A/chuva $(30 \mathrm{~mm})$ & $88,8 \mathrm{a}$ & 5 & $100,0 \mathrm{a}$ & 6 & $99,8 \mathrm{a}$ & 6 & $100,0 \mathrm{a}$ & 6 & $99,3 \mathrm{a}$ & 6 \\
\hline 7. Testemunha sem palha & $0,0 \mathrm{~d}$ & - & $0,0 \mathrm{~d}$ & - & $0,0 \mathrm{c}$ & - & $0,0 \mathrm{~d}$ & - & $0,0 \mathrm{c}$ & - \\
\hline 8. Testemunha com palha & $0,0 \mathrm{~d}$ & - & $0,0 \mathrm{~d}$ & - & $0,0 \mathrm{c}$ & - & $0,0 \mathrm{~d}$ & - & $0,0 \mathrm{c}$ & - \\
\hline $\mathrm{F}$ tratamento & \multicolumn{2}{|c|}{$234,31^{* *}$} & \multicolumn{2}{|c|}{$189,53^{* *}$} & \multicolumn{2}{|c|}{$785,91^{* *}$} & \multicolumn{2}{|c|}{$324,53 * *$} & \multicolumn{2}{|c|}{$74,48^{* *}$} \\
\hline $\mathrm{CV}(\%)$ & \multicolumn{2}{|c|}{11,01} & \multicolumn{2}{|c|}{9,21} & \multicolumn{2}{|c|}{4,45} & \multicolumn{2}{|c|}{6,97} & \multicolumn{2}{|c|}{15,88} \\
\hline DMS & \multicolumn{2}{|c|}{13.75} & \multicolumn{2}{|c|}{14.64} & \multicolumn{2}{|c|}{7,40} & \multicolumn{2}{|c|}{11.33} & \multicolumn{2}{|c|}{23,79} \\
\hline
\end{tabular}

Médias seguidas de mesma letra na coluna não diferem entre si pelo teste de Tukey $(\mathrm{P}<0,05)$. ${ }^{* *}$ - significativo a $1 \%$ de probabilidade. Obs.: A = aplicação de 880 e $220 \mathrm{~g} \mathrm{ha}^{-1}$ dos ingredientes ativos clomazone e hexazinone, respectivamente; $\mathrm{P}=$ cobertura com $5 \mathrm{tha}{ }^{-1}$ de palha de cana-de-açúcar. 
Os tratamentos em que o herbicida foi aplicado sobre a palha seca e com ausência de precipitação posterior (tratamentos 2 e 4) apresentaram controle insatisfatório para esta espécie. Os valores médios variaram de 36 a $57 \%$ e 15 a $55 \%$, para os tratamentos com aplicação do herbicida sobre a palha seca e sobre a palha umedecida 12 horas antes da pulverização, respectivamente. Com essas médias, esses tratamentos foram estatisticamente inferiores aos demais, desde o início das avaliações (Tabela 2). A presença da palha sem a aplicação do herbicida (tratamento 8) não influenciou a emergência das espécies estudadas.

Observando os dados de controle da espécie I. hederifolia (Tabela 6), nota-se que no início das avaliações (6 DAA) os melhores resultados são observados nos tratamentos 3 , em uma simulação à pulverização combinada com a colheita. Aos 13 DAA houve alto fluxo de germinação da espécie $I$. hederifolia no tratamento 5 , onde este, após 21 DAA, se restabeleceu, controlando assim a espécie em $98 \%$ (nota 6). De modo geral, os tratamentos com a aplicação do herbicida, na ausência ou presença de palha, e posterior chuva e/ou umidade do solo apresentaram controle total dessa espécie até 35 DAA - período considerado importante no desenvolvimento da cultura da cana-de-açúcar e crítico quando na presença de plantas daninhas.

Como observado para a espécie $B$. decumbens, os tratamentos com aplicação do herbicida sobre a palha seca e ausência de posterior precipitação que proporcionasse a retirada do produto da palha foram os menos eficientes no controle de I. hederifolia. Em todas as épocas de avaliação, esses tratamentos foram significativamente inferiores quando comparados aos demais tratamentos testados.

Considerando a espécie I. grandifolia, os resultados de controle pela mistura formulada foram considerados diferentes dos obtidos para I. hederifolia, conforme observado na Tabela 7, com excelente controle da espécie até 35 DAA, independentemente do tratamento empregado. Apesar de serem do mesmo gênero, o comportamento dos tratamentos nesta planta daninha foi bastante satisfatório. Apesar de o tratamento com precipitação 12 horas antes da pulverização (tratamento 4) apresentar menor eficiência no controle desta espécie no início das avaliações (nota 4; 71,3\%), ele respondeu bem no decorrer do experimento, com excelente controle (nota 6;97\%) aos

Tabela 6 - Porcentagem média e notas segundo a escala da ALAM de controle de Ipomoea hederifolia, pela mistura formulada de clomazone + hexazinone, aplicada em diferentes posicionamentos em relação à palha de cana-de-açúcar e à simulação de chuva. Botucatu-SP, 2007

\begin{tabular}{|c|c|c|c|c|c|c|c|c|c|c|}
\hline \multirow{3}{*}{ Tratamento } & \multicolumn{10}{|c|}{ Dias após a aplicação } \\
\hline & \multicolumn{2}{|c|}{6} & \multicolumn{2}{|c|}{13} & \multicolumn{2}{|c|}{21} & \multicolumn{2}{|c|}{27} & \multicolumn{2}{|c|}{35} \\
\hline & $\%$ & ALAM & $\%$ & ALAM & $\%$ & ALAM & $\%$ & ALAM & $\%$ & ALAM \\
\hline 1. $\mathrm{P} / \mathrm{A} / \mathrm{chuva}(30 \mathrm{~mm}-1 \mathrm{DAA})$ & $76,3 \mathrm{c}$ & 4 & $98,5 \mathrm{a}$ & 6 & $99,5 \mathrm{a}$ & 6 & $100,0 \mathrm{a}$ & 6 & $100,0 \mathrm{a}$ & 6 \\
\hline 2. Chuva $(30 \mathrm{~mm}) / \mathrm{P} / \mathrm{A}$ & $43,8 \mathrm{~d}$ & 2 & $46,3 \mathrm{~b}$ & 2 & $72,5 \mathrm{~b}$ & 4 & $84,5 \mathrm{~b}$ & 5 & $80,0 \mathrm{~b}$ & 4 \\
\hline 3. A/P & $96,3 \mathrm{ab}$ & 6 & $99,0 \mathrm{a}$ & 6 & $99,5 \mathrm{a}$ & 6 & $100,0 \mathrm{a}$ & 6 & $100,0 \mathrm{a}$ & 6 \\
\hline 4. $\mathrm{P} /$ chuva $(30 \mathrm{~mm}) / \mathrm{A}(12 \mathrm{~h}$ após $)$ & $13,8 \mathrm{e}$ & 1 & $38,8 \mathrm{~b}$ & 1 & $70,0 \mathrm{~b}$ & 3 & $49,3 \mathrm{c}$ & 2 & $32,5 \mathrm{c}$ & 1 \\
\hline 5. P/A/chuva $(2,5 \mathrm{~mm})$ & $100,0 \mathrm{a}$ & 6 & $46,3 \mathrm{~b}$ & 6 & $98,0 \mathrm{a}$ & 6 & $96,3 \mathrm{a}$ & 6 & $100,0 \mathrm{a}$ & 6 \\
\hline 6. A/chuva $(30 \mathrm{~mm})$ & $88,0 \mathrm{~b}$ & 5 & $97,3 \mathrm{a}$ & 6 & $99,5 \mathrm{a}$ & 6 & $99,3 \mathrm{a}$ & 6 & $100,0 \mathrm{a}$ & 6 \\
\hline 7. Testemunha sem palha & $0,0 \mathrm{f}$ & - & $0,0 \mathrm{c}$ & - & $0,0 \mathrm{c}$ & - & $0,0 \mathrm{~d}$ & - & $0,0 \mathrm{~d}$ & - \\
\hline 8. Testemunha com palha & $0,0 \mathrm{f}$ & - & $0,0 \mathrm{c}$ & - & $0,0 \mathrm{c}$ & - & $0,0 \mathrm{~d}$ & - & $0,0 \mathrm{~d}$ & - \\
\hline $\mathrm{F}$ tratamento & \multicolumn{2}{|c|}{$308,86^{* *}$} & \multicolumn{2}{|c|}{$104,64 * *$} & \multicolumn{2}{|c|}{$113,96^{* *}$} & \multicolumn{2}{|c|}{$459,02 * *$} & \multicolumn{2}{|c|}{$163,72^{* *}$} \\
\hline CV $(\%)$ & \multicolumn{2}{|c|}{9,42} & \multicolumn{2}{|c|}{15,24} & \multicolumn{2}{|c|}{12,05} & \multicolumn{2}{|c|}{6,24} & \multicolumn{2}{|c|}{11,15} \\
\hline DMS & \multicolumn{2}{|c|}{11,53} & \multicolumn{2}{|c|}{19,00} & \multicolumn{2}{|c|}{19,01} & \multicolumn{2}{|c|}{9,67} & \multicolumn{2}{|c|}{16,73} \\
\hline
\end{tabular}

Médias seguidas de mesma letra na coluna não diferem entre si pelo teste de Tukey $(\mathrm{P}<0,05)$. ** - significativo a $1 \%$ de probabilidade. Obs.: A = aplicação de 880 e $220 \mathrm{~g} \mathrm{ha}^{-1}$ dos ingredientes ativos clomazone e hexazinone, respectivamente; $\mathrm{P}=$ cobertura com $5 \mathrm{tha}^{-1} \mathrm{de}$ palha de cana-de-açúcar. 
Tabela 7 - Porcentagem média e notas segundo a escala da ALAM de controle de Ipomoea grandifolia pela mistura formulada de clomazone + hexazinone, aplicada em diferentes posicionamentos em relação à palha de cana-de-açúcar e à simulação de chuva. Botucatu-SP, 2007

\begin{tabular}{|c|c|c|c|c|c|c|c|c|c|c|}
\hline \multirow{3}{*}{ Tratamento } & \multicolumn{10}{|c|}{ Dias após a aplicação } \\
\hline & \multicolumn{2}{|c|}{6} & \multicolumn{2}{|c|}{13} & \multicolumn{2}{|c|}{21} & \multicolumn{2}{|c|}{27} & \multicolumn{2}{|c|}{35} \\
\hline & $\%$ & ALAM & $\%$ & ALAM & $\%$ & ALAM & $\%$ & ALAM & $\%$ & ALAM \\
\hline 1. P/A/chuva (30 mm - 1 DAA $)$ & $96,5 \mathrm{a}$ & 6 & $99,5 \mathrm{a}$ & 6 & $100,0 \mathrm{a}$ & 6 & $100,0 \mathrm{a}$ & 6 & $100,0 \mathrm{a}$ & 6 \\
\hline 2. Chuva $(30 \mathrm{~mm}) / \mathrm{P} / \mathrm{A}$ & $90,3 \mathrm{a}$ & 5 & $95,8 \mathrm{ab}$ & 6 & $100,0 \mathrm{a}$ & 6 & $99,3 \mathrm{ab}$ & 6 & $99,8 \mathrm{ab}$ & 6 \\
\hline 3. A/P & $98,8 \mathrm{a}$ & 6 & $100,0 \mathrm{a}$ & 6 & $99,8 \mathrm{a}$ & 6 & $99,8 \mathrm{a}$ & 6 & $100,0 \mathrm{a}$ & 6 \\
\hline 4. $\mathrm{P} /$ chuva $(30 \mathrm{~mm}) / \mathrm{A}(12 \mathrm{~h}$ após $)$ & $71,3 \mathrm{~b}$ & 4 & $91,8 \mathrm{~b}$ & 5 & $95,8 \mathrm{ab}$ & 6 & $97,5 \mathrm{~b}$ & 6 & $97,0 \mathrm{~b}$ & 6 \\
\hline 5. P/A/chuva $(2,5 \mathrm{~mm})$ & $100,0 \mathrm{a}$ & 6 & $95,5 \mathrm{ab}$ & 6 & $93,0 \mathrm{~b}$ & 6 & $100,0 \mathrm{a}$ & 6 & $98,8 \mathrm{ab}$ & 6 \\
\hline 6. A/chuva $(30 \mathrm{~mm})$ & $99,0 \mathrm{a}$ & 6 & $100,0 \mathrm{a}$ & 6 & $99,5 \mathrm{a}$ & 6 & $99,8 \mathrm{a}$ & 6 & $99,5 \mathrm{ab}$ & 6 \\
\hline 7. Testemunha sem palha & $0,0 \mathrm{c}$ & - & $0,0 \mathrm{c}$ & - & $0,0 \mathrm{c}$ & - & $0,0 \mathrm{c}$ & - & $0,0 \mathrm{c}$ & - \\
\hline 8. Testemunha com palha & $0,0 \mathrm{c}$ & - & $0,0 \mathrm{c}$ & - & $0,0 \mathrm{c}$ & - & $0,0 \mathrm{c}$ & - & $0,0 \mathrm{c}$ & - \\
\hline $\mathrm{F}$ tratamento & \multicolumn{2}{|c|}{$384,12 * *$} & \multicolumn{2}{|c|}{$1.016,45^{* *}$} & \multicolumn{2}{|c|}{$1.896,29 * *$} & \multicolumn{2}{|c|}{$9.563,00 * *$} & \multicolumn{2}{|c|}{$5.256,93 * *$} \\
\hline $\mathrm{CV}(\%)$ & \multicolumn{2}{|c|}{6,45} & \multicolumn{2}{|c|}{3,88} & \multicolumn{2}{|c|}{2,84} & \multicolumn{2}{|c|}{1,26} & \multicolumn{2}{|c|}{1,70} \\
\hline DMS & \multicolumn{2}{|c|}{10,49} & \multicolumn{2}{|c|}{6,66} & \multicolumn{2}{|c|}{4,88} & \multicolumn{2}{|c|}{2,20} & \multicolumn{2}{|c|}{2,96} \\
\hline
\end{tabular}

Médias seguidas de mesma letra na coluna não diferem entre si pelo teste de Tukey $(\mathrm{P}<0,05)$. ** - significativo a $1 \%$ de probabilidade. Obs.: $\mathrm{A}=$ aplicação de 880 e $220 \mathrm{~g} \mathrm{ha}^{-1}$ dos ingredientes ativos clomazone e hexazinone, respectivamente; $\mathrm{P}=$ cobertura com $5 \mathrm{t}^{-1} \mathrm{de}^{-}$ palha de cana-de-açúcar.

35 DAA. Negrisoli et al. (2009) e Toledo et al. (2009) observaram resultados semelhantes, com excelente controle de I. grandifolia com a aplicação dos herbicidas oxyfluorfen e amicarbazone sob diferentes manejos da palha da cana-de-açúcar.

Para a espécie E. heterophylla (Tabela 8), os resultados obtidos pela mistura formulada apresentaram certa particularidade. No início das avaliações (6 DAA), observou-se controle total da espécie pelos tratamentos propostos, quando comparados às testemunhas. A partir disso, ocorreu perda da eficácia de alguns tratamentos até o final das avaliações. Nota-se que menores médias de controle são encontradas no tratamento com aplicação em palha úmida, sem ocorrência de chuva posterior (tratamento 4), indicando que não ocorreu a saída do produto para o solo, nem o simples contato da plântula, durante sua emergência, com a palha impregnada com o herbicida foi suficiente para determinar o controle desta espécie. Resultado semelhante ao deste tratamento foi observado no tratamento com ausência de palha, que apresentou média de 99,3\% (nota 6) no final das avaliações.

O tratamento com aplicação em palha seca e sem a ocorrência de chuva posterior (tratamento 2), apesar de apresentar médias em torno de $85 \%$ aos 13 e 21 DAA, mostrou-se eficaz a partir de 27 DAA, chegando a 95\% (nota 6) de controle desta espécie ao final das avaliações.

Quanto aos demais tratamentos, onde há ocorrência de precipitação posterior à aplicação de herbicida, os resultados foram bastante satisfatórios, proporcionando excelente controle (médias acima de 98\%) de E. heterophylla. Monquero et al. (2009) observaram que os tratamentos aplicados em pré-emergência dos herbicidas trifloxysulfuron-sodium + ametryn, mesotrione e metribuzin foram eficazes no controle de Euphorbia heterophylla e Ipomoea grandifolia, com excelente controle (nota 6; > 90\%) das espécies estudadas aos 90 DAA.

Independentemente da planta daninha estudada, os tratamentos com maiores porcentagens de controle aos 35 DAA foram aqueles em que o herbicida testado atingiu o solo, seja através da aplicação direta ou da lixiviação do herbicida, promovida pela simulação da chuva após sua aplicação. Esses resultados mostram que um volume de chuva de apenas $2,5 \mathrm{~mm}$ após a aplicação do herbicida sobre a palha pode promover a lixiviação do produto para o solo e favorecer a absorção do herbicida pelas espécies estudadas. 
Tabela 8 - Porcentagem média e notas segundo a escala da ALAM de controle de Euphorbia heterophylla pela mistura formulada de clomazone + hexazinone, aplicada em diferentes posicionamentos em relação à palha de cana-de-açúcar e à simulação de chuva. Botucatu-SP, 2007

\begin{tabular}{|c|c|c|c|c|c|c|c|c|c|c|}
\hline \multirow{3}{*}{ Tratamento } & \multicolumn{10}{|c|}{ Dias após a aplicação } \\
\hline & \multicolumn{2}{|c|}{6} & \multicolumn{2}{|c|}{13} & \multicolumn{2}{|c|}{21} & \multicolumn{2}{|c|}{27} & \multicolumn{2}{|c|}{35} \\
\hline & $\%$ & ALAM & $\%$ & ALAM & $\%$ & ALAM & $\%$ & ALAM & $\%$ & ALAM \\
\hline 1. P/A/chuva (30 mm - 1 DAA) & $100,0 \mathrm{a}$ & 6 & $100,0 \mathrm{a}$ & 6 & $96,3 \mathrm{a}$ & 6 & $100,0 \mathrm{a}$ & 6 & $100,0 \mathrm{a}$ & 6 \\
\hline 2. Chuva $(30 \mathrm{~mm}) / \mathrm{P} / \mathrm{A}$ & $100,0 \mathrm{a}$ & 6 & $85,0 \mathrm{ab}$ & 5 & $87,5 \mathrm{a}$ & 5 & $100,0 \mathrm{a}$ & 6 & $95,0 \mathrm{a}$ & 6 \\
\hline 3. A/P & $100,0 \mathrm{a}$ & 6 & $100,0 \mathrm{a}$ & 6 & $100,0 \mathrm{a}$ & 6 & $100,0 \mathrm{a}$ & 6 & $100,0 \mathrm{a}$ & 6 \\
\hline 4. $\mathrm{P} /$ chuva $(30 \mathrm{~mm}) / \mathrm{A}(12 \mathrm{~h}$ após $)$ & $100,0 \mathrm{a}$ & 6 & $75,0 \mathrm{~b}$ & 4 & $67,5 \mathrm{a}$ & 3 & $75,0 \mathrm{a}$ & 4 & $75,0 \mathrm{a}$ & 4 \\
\hline 5. P/A/chuva $(2,5 \mathrm{~mm})$ & $100,0 \mathrm{a}$ & 6 & $98,0 \mathrm{a}$ & 6 & $99,3 \mathrm{a}$ & 6 & $100,0 \mathrm{a}$ & 6 & $98,5 \mathrm{a}$ & 6 \\
\hline 6. A/chuva $(30 \mathrm{~mm})$ & $100,0 \mathrm{a}$ & 6 & $98,3 \mathrm{a}$ & 6 & $98,3 \mathrm{a}$ & 6 & $99,3 \mathrm{a}$ & 6 & $99,3 \mathrm{a}$ & 6 \\
\hline 7. Testemunha sem palha & $0,0 \mathrm{~b}$ & - & $0,0 \mathrm{c}$ & - & $0,0 \mathrm{~b}$ & - & $0,0 \mathrm{~b}$ & - & $0,0 \mathrm{~b}$ & - \\
\hline 8. Testemunha com palha & $0,0 \mathrm{~b}$ & - & $0,0 \mathrm{c}$ & - & $0,0 \mathrm{~b}$ & - & $0,0 \mathrm{~b}$ & - & $0,0 \mathrm{~b}$ & - \\
\hline F tratamento & \multicolumn{2}{|l|}{-} & \multicolumn{2}{|c|}{$126,96 * *$} & \multicolumn{2}{|c|}{$24,27 * *$} & \multicolumn{2}{|c|}{$26,04 * *$} & \multicolumn{2}{|c|}{$22,91 * *$} \\
\hline $\mathrm{CV}(\%)$ & \multicolumn{2}{|c|}{-} & \multicolumn{2}{|c|}{11,18} & \multicolumn{2}{|c|}{25,83} & \multicolumn{2}{|c|}{24,64} & \multicolumn{2}{|c|}{26,42} \\
\hline DMS & \multicolumn{2}{|c|}{-} & \multicolumn{2}{|c|}{18,21} & \multicolumn{2}{|c|}{41,51} & \multicolumn{2}{|c|}{41,00} & \multicolumn{2}{|c|}{42,39} \\
\hline
\end{tabular}

Médias seguidas de mesma letra na coluna não diferem entre si pelo teste de Tukey $(\mathrm{P}<0,05)$. ** - significativo a $1 \%$ de probabilidade Obs.: A = aplicação de 880 e $220 \mathrm{~g} \mathrm{ha}^{-1}$ dos ingredientes ativos clomazone e hexazinone, respectivamente; $\mathrm{P}=$ cobertura com $5 \mathrm{tha}^{-1}$ de palha de cana-de-açúcar.

\section{LITERATURA CITADA}

AZANIA, A. A. P. M. et al. Interferência de palha de cana-deaçúcar (Saccharum spp.) na emergência das espécies de plantas daninhas da família Convolvulaceae.

Planta Daninha, v. 20, n. 1, p. 207-212, 2002.

ASOCIACIÓN LATINOAMERICANA DE MALEZAS ALAM. Recomendaciones sobre unificación de los sistemas de evaluación en ensayos de control de malezas. ALAM, v. 1, n. 1, p. $35-38,1974$

BANKS, P. A.; ROBINSON, E. L. The influence of straw mulch on the soil reception and persistence of metribuzin Weed Sci., v. 30, n. 2, p. 164-168, 1982.

BANKS, P. A.; ROBINSON, E. L. The fate of oryzalin applied to straw-mulched and non-mulched soils. Weed Sci., v. 32, p. $269-272,1984$

BANKS, P. A.; ROBINSON, E. L. Soil reception and activity of acetochlor, alachlor and metolachlor as affected by wheat (Triticum aestivum), atraw irrigation. Leed Sci., v. 34, p. 607-611, 1986.

CORREA, M. M.; DURIGAN, J. C. Emergência de plantas daninhas em solo coberto com palha de cana-de-açúcar.

Planta Daninha, v. 22, n. 1, p. 11-17, 2004.

DEUBER, R. Ciência das plantas daninhas: fundamentos. Jaboticabal: FUNEP, 1992. v. 1. 431 p.
DURIGAN, J. C.; TIMOSSI, P. C.; CORREIA, N. M. Manejo integrado da tiririca na produtividade de cana-deaçúcar. Planta Daninha, v. 24, n. 1, p. 77-81, 2006.

GRAVENA, R. et al. Controle de plantas daninhas através da palha de cana-de-açúcar associada à mistura dos herbicidas trifloxysulfuron sodium + ametrina. Planta Daninha, v. 22, n. 3, p. 419-427, 2004.

KUVA, M.; PITELLI, R. A.; SALGADO, T. P.

Fitossociologia de comunidades de plantas daninhas em agroecossistema cana-crua. Planta Daninha, v. 25, n. 3, p. 501-511, 2007.

LAMOREAUX, R. J.; JAIN, R.; HESS, F. D. Efficacy of dimethenamid, metolachlor and encapsulated alachlor in soil covered with crop residue. Bringhton Crop Protec. Conf. Weeds, v. 3, p. 1015-1020, 1993.

MARTINS, D. et al. Emergência em campo de dicotiledôneas infestantes em solo coberto com palha de cana-de-açúcar. Planta Daninha, v.17, n. 1, p. 151-161, 1999.

MONQUERO, P. A. et al. Eficácia de herbicidas aplicados em diferentes épocas e espécies daninhas em área de cana-deaçúcar colhida mecanicamente. Planta Daninha, v. 27, n. 2, p.309-317, 2009

NEGRISOLI, E. et al. Eficácia do herbicida oxyfluorfen com a cobertura de palha no controle de plantas daninhas.

Planta Daninha, v. 27, n. 1, p. 197-203, 2009. 
NEGRISOLI, E. et al. Associação do herbicida tebuthiuron com a cobertura de palha no controle de plantas daninhas no sistema de cana-crua. Planta Daninha, v. 25, n. 3, p. 621-628, 2007a.

NEGRISOLI, E. et al. Controle de plantas daninhas pelo amicarbazone aplicado na presença de palha de cana-deaçúcar. Planta Daninha, v. 25, n. 3, p. 603-611, 2007 b.

NEGRISOLI, E. et al. Depossition and leaching of tebuthiuron on sugar cane straw applied with and without alkkyl poyglycoside adjuvnt. J. Environ. Sci. Health. Part B, Pestic. Food Contam. Agric. Wastes, v. B40, n. 1, p. 207-214, 2005.

OLIVEIRA, A. R.; FREITAS, S. P. Levantamento fitossociológico de plantas daninhas em áreas de produção de cana-de-açúcar. Planta Daninha, v. 26, n. 1, p. 33-46, 2008.
RODRIGUES, B. N.; ALMEIDA, F. S. Guia de herbicidas. 5.ed. Londrina: Edição do Autores, 2005. 591 p.

SOCIEDADE BRASILEIRA DA CIÊNCIA DAS PLANTAS DANINHAS - SBCPD. Procedimentos para instalação, avaliação e análise de experimentos com herbicidas. Londrina: 1995. $42 \mathrm{p}$

TOLEDO, R. E. B. et al. Eficácia do herbicida amicarbazone aplicado sobre a palha ou no solo no controle de plantas daninhas na cultura da cana-de-açúcar. Planta Daninha, v. 27, n. 2 , p. $319-326,2009$

VELINI, E. D.; NEGRISOLI, E. Controle de plantas daninhas em cana crua. In: CONGRESSO BRASILEIRO DA CIENNCIA DAS PLANTAS DANINHAS, 22., 2000, Foz de Iguaçu. Palestras... Foz de Iguaçu: Sociedade Brasileira da Ciência das Plantas Daninhas, 2000. p. 148-164. 